\title{
Resultate der Umfrage 2014 zur Beurteilung der Weiterbildung
}

\author{
Bernadette Sütterlina, Lea Christina Burgermeister ${ }^{b}$, Michael Siegrist $^{c}$, Werner Bauer ${ }^{d}$ \\ ${ }^{a}$ Dr. sc., Senior Researcher; ${ }^{b}$ Lic. phil., wissenschaftliche Mitarbeiterin; ${ }^{c}$ Prof. Dr. phil., Professor für Consumer Behavior an der ETH Zürich; \\ d Dr. med., Präsident SIWF
}

Unter dem Mandat des Bundes verleiht das Schweizerische Institut für ärztliche Weiter- und Fortbildung (SIWF) die Facharzttitel und ist zusammen mit den Fachgesellschaften für die Gestaltung der Weiterbildung verantwortlich. Auch wenn die Weiterbildung einen sehr guten Ruf geniesst, muss ihre Qualität kontinuierlich evaluiert werden. Dies geschieht im Rahmen der Beurteilung des Weiterbildungskonzepts jeder einzelnen Weiterbildungsstätte, sodann mit Hilfe der Visitationen vor Ort und der jährlichen Umfrage bei den Ärztinnen und Ärzten in Weiterbildung. Im Jahr 2014 wurde die Umfrage im Auftrag des SIWF bereits zum 18. Mal durchgeführt.

Jede Leiterin und jeder Leiter einer anerkannten Weiterbildungsstätte erhielt im November 2014 einen Bericht mit der detaillierten Rückmeldung zu den Ergebnissen der Weiterbildungsstätte. Im Bericht wird zudem als Vergleichswert der Durchschnitt der Beurteilungen aller Weiterbildungsstätten der entsprechenden Fachrichtung abgebildet (bei den grössten Fachrichtungen wird bei der Berechnung des Vergleichswertes zudem nach Grösse der Weiterbildungsstätte differenziert). Eine Übersicht der Ergebnisse (Spinnengrafik) der einzelnen Weiterbildungsstätten wird jeweils auf der SIWF-Website publiziert.

Ziel dieser jährlichen Umfrage ist es, zur Sicherstellung der Qualität der Weiterbildung beizutragen. Dabei werden acht Beurteilungsdimensionen erfasst: Globalbeurteilung, Vermittlung von Fachkompetenz, Lernkultur, Führungskultur, Fehlerkultur, Entscheidungskultur, Betriebskultur sowie Evidence based medicine. Neben diesem allgemeinen Teil, der von Jahr zu Jahr weitgehend gleich bleibt, beinhaltet der Fragebogen jedes Jahr einen variablen Modulteil. In diesem werden die Ärztinnen und Ärzte zu aktuellen Themen mit Bezug zu ihrer Weiterbildung befragt. Im Jahr 2014 umfasste dieser Modulteil Fragen zu den Auswirkungen der flächendeckenden Einführung des SwissDRGSystems sowie zu den Determinanten der Wahl des Facharzttitels. Fragen zum DRG-System waren bereits vor dessen Einführung in der Umfrage 2011 gestellt worden. Die erneute Befragung zu diesem Thema im Jahr 2014 ermöglichte somit einen direkten Vergleich der erwarteten Auswirkungen vor der Einführung des DRG-Systems mit den wahrgenommenen Veränderungen nach der Einführung.

Zur Vorbereitung der Befragung findet jeweils im Frühling eine statistische Erhebung der Weiterbildungsstellen statt, in der die Leiterinnen und Leiter der Weiterbildungsstätten Angaben zu den bei ihnen angestellten Weiterzubildenden machen. Im Rahmen dieser Erhebung werden auch die Leiterinnen und Leiter zusätzlich zu aktuellen Themen befragt. Analog zur Befragung der Ärztinnen und Ärzte in Weiterbildung umfasste auch der Fragebogen für die Leiterinnen und Leiter der Weiterbildungsstätten im Jahr 2014 Fragen zu den Auswirkungen der flächendeckenden Einführung des SwissDRG-Systems. Diese waren ebenfalls bereits Teil der Befragung der Leiterinnen und Leiter im Jahr 2011.

Im vorliegenden Artikel geben wir einen Überblick über die wichtigsten Ergebnisse der Umfrage 2014; wenn möglich werden zusätzlich Vergleichszahlen aus der Umfrage 2011 präsentiert.

\section{Methode}

\section{Umfrageteilnehmer und Durchführung}

An der Befragung 2014 nahmen 1275 Weiterbildungsstätten teil und es wurden insgesamt 10328 Fragebogen für die Ärztinnen und Ärzte in Weiterbildung verschickt. Die Leiterinnen und Leiter der Weiterbildungsstätten waren für die Verteilung der zugeschickten Fragebogen an die von ihnen gemeldeten Ärztinnen und Ärzte in Weiterbildung zuständig. Die ausgefüllten Fragebogen wurden in einem individuellen Rückantwortcouvert anonym direkt an die Professur Consumer Behavior der ETH Zürich retourniert, die mit der Durchführung der jährlichen Befragung betraut ist. Die Datenerhebung umfasste einen Zeitraum von ungefähr drei Monaten (Anfang Juli bis Mitte Oktober 2014). Die Rücklaufquote betrug $66 \%$ $(\mathrm{N}=6831)$. Dieser Wert ist vergleichbar mit der Rücklaufquote früherer Umfragen [1]. 
Im Zusammenhang mit der statistischen Erhebung der Weiterbildungsstellen im Frühling wurde den Leiterinnen und Leitern der Weiterbildungsstätten ein Link zu einem kurzen Online-Fragebogen zugeschickt. Dieser wurde von 1370 Leiterinnen und Leitern ausgefüllt (94\%). Für die Analyse in Bezug auf die nachfolgend präsentierten Ergebnisse wurden nur die Antworten der Leiterinnen und Leiter der Weiterbildungsstätten einbezogen, die angaben, mit dem SwissDRG-System zu arbeiten $(\mathrm{N}=1008)$.

\section{Fragebogen}

Der Fragebogen für die Ärztinnen und Ärzte in Weiterbildung war in drei Sprachversionen verfügbar. Die Fragen zu den Beurteilungsdimensionen, Globalbeurteilung, Lernkultur, Führungskultur, Fehlerkultur, Entscheidungskultur, Betriebskultur sowie Evidence based medicine wurden in Form von Aussagen formuliert. Die Teilnehmer wurden gebeten, anzugeben, inwiefern sie eine Aussage als zutreffend betrachten. Beim Frageteil zu den Fachkompetenzen wurden die konkreten Kompetenzen aufgelistet und die Teilnehmer beurteilten, wie gross der Beitrag der Weiterbildungsstätte zu deren Entwicklung ist.

Der jährlich wechselnde Modulteil beinhaltete ein Modul mit Fragen zur flächendeckenden Einführung der neuen Spitalfinanzierung/DRG und ein Modul mit Fragen zur Wahl der Facharztrichtung. Beim DRG-Modul wurde die Meinung der Ärztinnen und Ärzte in Weiterbildung zu den Auswirkungen des DRG-Systems auf ihre Arbeit erhoben. Zudem wurde gefragt, inwiefern das DRG-System aus ihrer Sicht den einzelnen Patientengruppen gerecht wird. Die Fragen wurden in Form von Aussagen formuliert und die Teilnehmer wurden gebeten, ihre Zustimmung anzugeben. Einige Fragen zur neuen Spitalfinanzierung/DRG wurden bereits vor der flächendeckenden Einführung in der Umfrage 2011 gestellt. Dies ermöglichte entsprechend

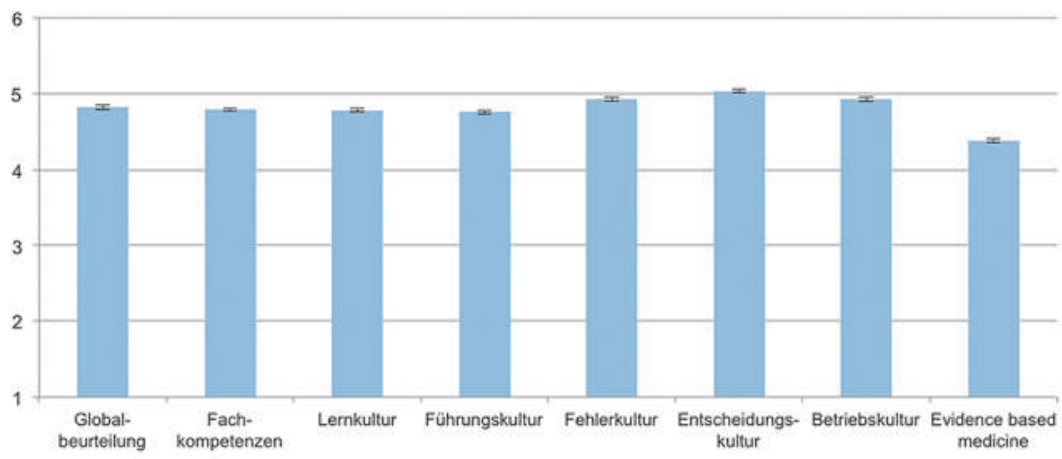

Abbildung 1: Mittelwerte (inkl. 95\%-Konfidenzintervall) der acht Beurteilungsdimensionen (je höher der Wert, desto besser die Beurteilung). Nur die Antworten von Ärztinnen und Ärzten in Weiterbildung, die 2 Monate oder länger an der Weiterbildungsstätte tätig waren, wurden berücksichtigt. einen Vergleich der Antworten der Ärztinnen und Ärzte in Weiterbildung vor der Einführung des DRGSystems mit den Antworten nach der Einführung. Analog zu der Befragung der Ärztinnen und Ärzte in Weiterbildung wurden in den Jahren 2011 und 2014 im Rahmen der statistischen Erhebung auch den Leiterinnen und Leitern der Weiterbildungsstätten entsprechende Fragen zu den Auswirkungen der Einführung des DRG-Systems gestellt.

Das Modul zur Wahl der Fachrichtung beinhaltete zum einen Fragen zu den Beweggründen für die Wahl. Es wurden zehn unterschiedliche Aspekte aufgelistet, und die Ärztinnen und Ärzte in Weiterbildung stuften jeweils ein, wie stark der entsprechende Aspekt ihre Wahl der Fachrichtung beeinflusst/beeinflusste. Der zweite Teil des Moduls zur Fachrichtungswahl befasste sich mit dem Zeitpunkt der Wahl. In einer ersten Frage machten die Teilnehmer Angaben dazu, wann sie die Wahl getroffen hatten, und in einer zweiten Frage wurde erfasst, ob sie die Fachrichtung während der Weiterbildung geändert hatten.

\section{Ergebnisse}

\section{Allgemeine Beurteilung der Weiterbildungs- stätten}

Insgesamt wird die Situation an den Weiterbildungsstätten von den Ärztinnen und Ärzten in Weiterbildung als gut beurteilt. Weiterbildungsstätten mit weniger Ärztinnen und Ärzten in Weiterbildung schneiden wie immer etwas besser ab [1]. In Abbildung 1 sind die acht unterschiedlichen Beurteilungsdimensionen dargestellt. Die Beurteilungen der Fehlerkultur, der Entscheidungskultur und der Betriebskultur fallen am besten aus, während der Bereich Evidence based medicine etwas abfällt.

\section{Neue Spitalfinanzierung/DRG}

\section{Angaben der Leiterinnen und Leiter der Weiterbildungsstätten}

Auswirkungen auf die Weiterbildung: Im Jahr 2011 waren 51\% der befragten Leiterinnen und Leiter der Meinung, dass an ihrer Weiterbildungsstätte durch die Einführung des DRG-Systems Weiterbildungsstellen gefährdet sind. Drei Jahre später gaben jedoch nur $4 \%$ an, infolge der Einführung des DRG-Systems tatsächlich Weiterbildungsstellen abgebaut zu haben. Auch bei der Frage, ob die Leiterinnen und Leiter sich aufgrund der Einführung des DRG-Systems überlegen, Ärztinnen/ Ärzte in Weiterbildung durch Spitalärztinnen/Spitalärzte zu ersetzen, stimmten vor der Einführung 29\% 


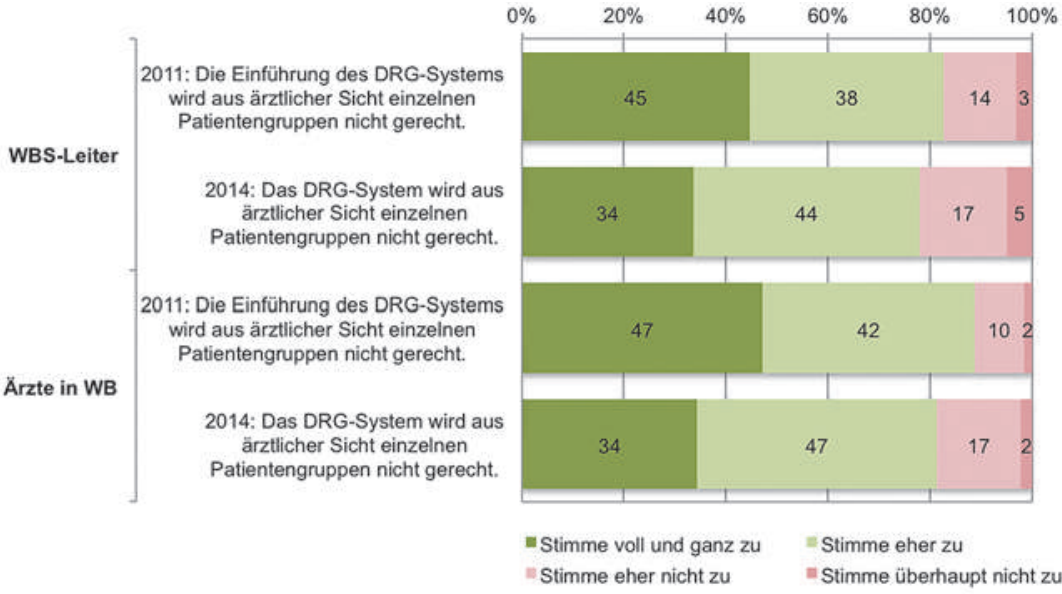

Abbildung 2: Überzeugung, dass das DRG-System aus ärztlicher Sicht einzelnen Patientengruppen nicht gerecht wird, vor und nach der Einführung des DRG-Systems, dargestellt für Leiterinnen und Leiter der Weiterbildungsstätten (2011: N = 1070; 2014: $N=1008$ ) und Ärztinnen und Ärzte in Weiterbildung (2011: $N=6281 ; 2014: N=6831$ ).

zu, während im Jahr 2014 nur 3\% bestätigten, dies effektiv umgesetzt zu haben. Allerdings gaben im Jahr 2014 doch 19\% an, dass sie sich überlegen, (weitere) Ärztinnen/Ärzte in Weiterbildung durch Spitalärztinnen/ Spitalärzte zu ersetzen.

Auswirkungen auf die Patienten: $\mathrm{Zu}$ den Auswirkungen des DRG-Systems auf die Patienten besteht seitens der Befragten ein klares Meinungsbild. Vor der Einführung des DRG-Systems vertrat die Mehrheit von $83 \%$ der Leiterinnen und Leiter die Meinung, dass das DRGSystem aus ärztlicher Sicht einzelnen Patientengruppen nicht gerecht wird. Diese Befürchtung scheint sich nach der Einführung leider tatsächlich zu bewahrheiten, denn 78\% der Befragten waren im Jahr 2014 immer noch dieser Auffassung. Das Meinungsbild blieb also im Wesentlichen unverändert.

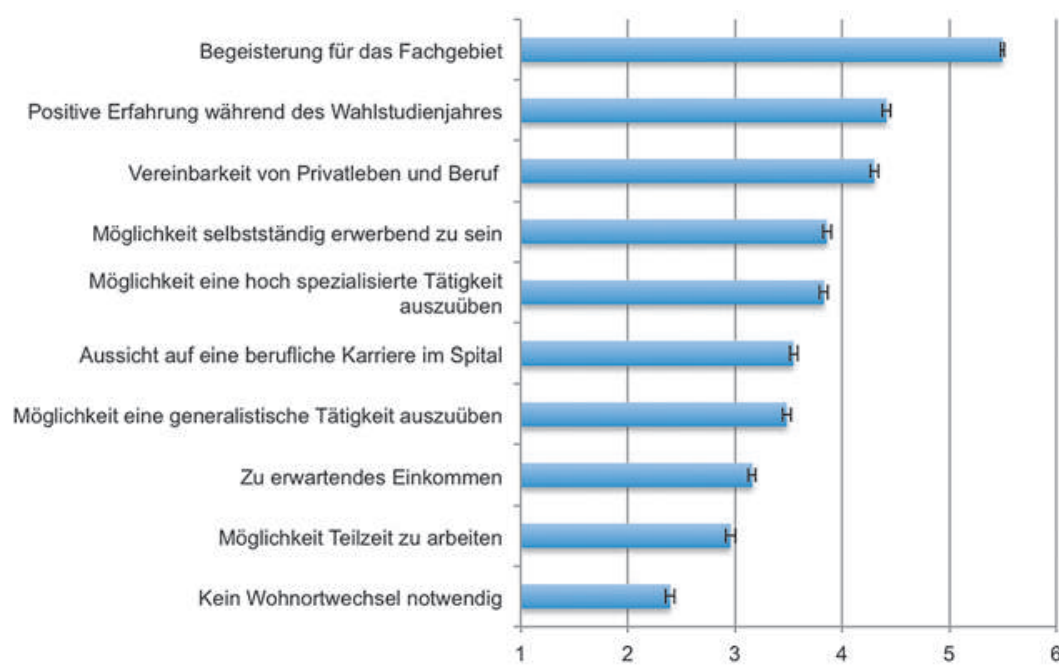

Abbildung 3: Einflussfaktoren bei der Wahl der Fachrichtung ( 1 = überhaupt kein Einfluss, $6=$ sehr starker Einfluss), sortiert nach Einflussstärke. Die Mittelwerte und die entsprechenden 95\%-Konfidenzintervalle sind ausgewiesen.

\section{Angaben der Ärztinnen und Ärzte in Weiterbildung}

Auswirkungen auf die Weiterbildung: Negativ, aber weniger negativ als befürchtet, scheinen die Auswirkungen der flächendeckenden Einführung der neuen Spitalfinanzierung/DRG im Hinblick auf die Weiterbildungssituation zu sein. Während im Jahr 2011 71\% der Ärztinnen und Ärzte in Weiterbildung befürchteten, dass sich die Einführung der neuen Spitalfinanzierung/DRG negativ auf ihre Arbeit auswirken wird, waren im Jahr 2014 noch 54\% dieser Überzeugung. Auswirkungen auf die Patienten: Mit einem Anteil von 89\% im Jahr 2011 und 81\% im Jahr 2014 ist die Überzeugung, dass das DRG-System aus ärztlicher Sicht einzelnen Patientengruppen nicht gerecht wird, unter den Ärztinnen und Ärzten in Weiterbildung sogar noch stärker verbreitet als unter den Leiterinnen und Leitern. Die detaillierten Ergebnisse sind in Abbildung 2 dargestellt.

\section{Wahl der Fachrichtung}

Beweggründe für die Wahl der Fachrichtung: Der mit Abstand wichtigste Aspekt bei der Entscheidung für eine Fachrichtung war die Begeisterung für das Fachgebiet. Aber auch positive Erfahrungen während des Wahlstudienjahres sowie die Vereinbarkeit von Privatleben und Beruf haben einen starken Einfluss. Hingegen spielen ein allfälliger Wohnortwechsel, die Möglichkeit, Teilzeit zu arbeiten, sowie das zu erwartende Einkommen eine geringere Rolle. Die Ergebnisse zu den Einflussfaktoren bei der Wahl des Facharzttitels sind in Abbildung 3 dargestellt.

In der Wichtigkeit der einzelnen Aspekte zeigen sich Unterschiede zwischen den Fachrichtungen. Werden die sieben häufigsten Disziplinen betrachtet, steht die Begeisterung für das Fachgebiet bei allen an erster Stelle. Auch das Wahlstudienjahr befindet sich, ausser bei der Allgemeinen Inneren Medizin und der Psychiatrie, auf einem der ersten drei Ränge. Ein Wohnortwechsel sowie das Einkommen werden bei allen Fachrichtungen, ausser der Orthopädischen Chirurgie, auf die hinteren drei Ränge verwiesen. Einzig bei der Orthopädischen Chirurgie wird das zu erwartende Einkommen als mittelstarker Einflussfaktor genannt. Die Wichtigkeit der Vereinbarkeit von Privatleben und Beruf variiert stark zwischen den verschiedenen Fachrichtungen. Während sie bei der Entscheidung für die Fachrichtungen Allgemeine Innere Medizin, Anästhesiologie und Psychiatrie einen wichtigen Faktor darstellt, ist sie für die Wahl der übrigen Fachrichtungen eher von mittlerer Relevanz. (Die detaillierten Angaben für die sieben häufigsten Fachrichtungen können online eingesehen werden unter www.saez.ch $\rightarrow$ Aktuelle Ausgabe $\rightarrow$ Tabelle 1.) 
Wann haben Sie sich für einen Facharzttitel entschieden?

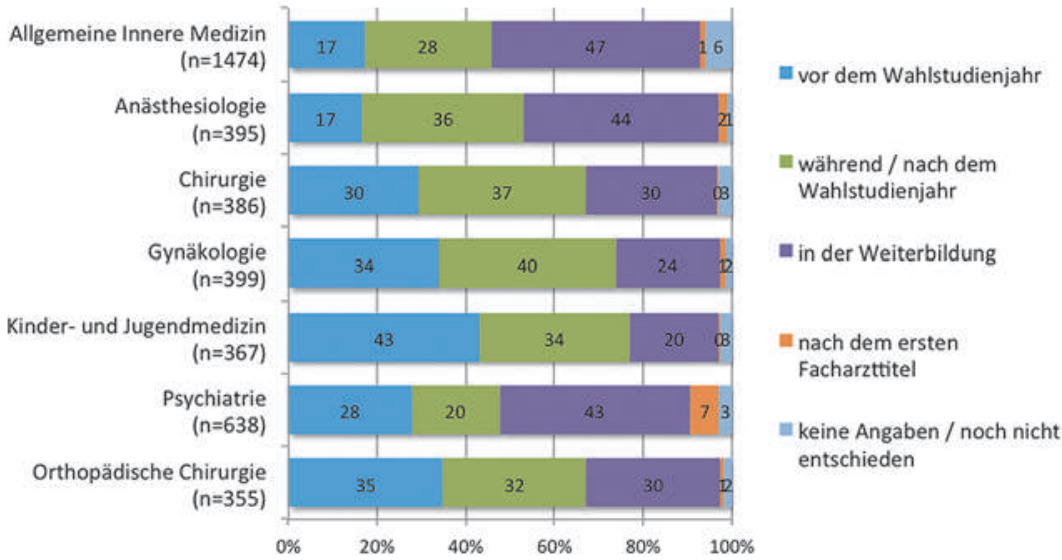

Abbildung 4: Zeitpunkt der Wahl einer Fachrichtung, differenziert nach den sieben am häufigsten angestrebten Fachrichtungen.

Zeitpunkt der Wahl der Fachrichtung: Die Entscheidung für eine Fachrichtung wurde lediglich bei der Hälfte der Weiterzubildenden bereits vor dem Beginn der Weiterbildung getroffen. Bei $28 \%$ fiel die Entscheidung während oder nach dem Wahlstudienjahr und 23\% entschieden sich bereits vor dem Wahlstudienjahr. Etwa ein Drittel (36\%) legte sich während der Weiterbildung für eine Fachrichtung fest und ein sehr kleiner Anteil (4\%) entschied sich erst nach dem ersten Facharzttitel. Die restlichen Umfrageteilnehmer hatten sich noch auf keine Fachrichtung festgelegt oder machten keine Angaben dazu. Der Zeitpunkt der Wahl variiert je nach angestrebtem Facharzttitel stark (Abb. 4). Für die Allgemeine Innere Medizin, die Anästhesiologie sowie die Psychiatrie entschieden sich die angehenden Fachärztinnen und Fachärzte mehrheitlich erst während der Weiterbildung. Auffallend ist, dass bei einem Grossteil der Anwärter auf den Facharzttitel in Kinder- und Jugendmedizin die Entscheidung bereits vor dem Wahlstudienjahr feststand.

\section{Diskussion}

Die Befragung 2014 zeigt, dass die grosse Mehrheit der an der Umfrage teilnehmenden Ärztinnen und Ärzte in Weiterbildung mit der vermittelten Weiterbildung insgesamt zufrieden ist und ihre Weiterbildungsstätte grundsätzlich positiv beurteilt. Die Umfrage ist für die Leiterinnen und Leiter der Weiterbildungsstätten ein wertvolles Tool für eine periodische Standort- bestimmung in Bezug auf die wahrgenommene Qualität der von ihnen angebotenen Weiterbildung. Sie hilft ihnen, allfällige Defizite zu identifizieren, problematische Entwicklungen bereits im Ansatz zu erkennen und gezielt Verbesserungsmassnahmen zu ergreifen. Die seltenen Fälle, die resultatmässig insgesamt klar im ungenügenden Bereich liegen, werden der jeweiligen Fachgesellschaft gemeldet, welche die Situation zu überprüfen und vor allem im Wiederholungsfall über allfällige Konsequenzen (z.B. eine Visitation oder Infragestellung der Anerkennung) zu entscheiden hat.

Im Hinblick auf eine bessere Planbar- und Steuerbarkeit des Angebots in den einzelnen Facharztgruppen zur Sicherstellung der künftigen ärztlichen Versorgung wird heute diskutiert, ob die angehenden Fachärztinnen und Fachärzte sich früher für einen Titel festlegen sollten. Die Auswertung der Angaben bezüglich des Zeitpunkts der Wahl der Fachrichtung zeigt, dass bei gewissen Fachrichtungen wie der Allgemeinen Inneren Medizin und der Psychiatrie diese Entscheidung relativ spät fällt. Es stellt sich daher die Frage, ob bei einer forcierten früheren Festlegung für eine Fachrichtung die Ärztinnen und Ärzte in Weiterbildung auch tatsächlich noch diejenige Fachrichtung wählen können, die langfristig am besten ihren Interessen und ihrer Eignung entspricht.

Bei der Einführung des DRG-Systems gab es viele Bedenken bezüglich der möglichen negativen Auswirkungen auf die Ausübung der ärztlichen Tätigkeit, die Weiterbildung und die Behandlung der Patienten. Wie die Umfrageergebnisse zeigen, hat die Einführung des DRG-Systems bis heute nicht so schwerwiegende negative Auswirkungen mit sich gebracht, wie sie vor der Einführung befürchtet worden waren. Es muss aber festgehalten werden, dass doch ein grosser Teil der Leiterinnen und Leiter der Weiterbildungsstätten und der Ärztinnen und Ärzte in Weiterbildung Nachteile des DRG-Systems bei der täglichen Arbeit erlebt und vor allem auch die bedenkliche Feststellung macht, dass das neue System einzelnen Patientengruppen nicht gerecht wird.

\section{Literatur}

1 Orlow P, Siegrist M, Giger M. Trends in der ärztlichen Weiterbildung. Die wichtigsten Resultate der Umfrage 2006 bei Assistenzärztinnen und Assistenzärzten. Schweiz Ärztezeitung. 2007;88(14/15):633-42. 